\title{
CDISC SDTM Observational Study Biospecimen Retention Terminology
}

National Cancer Institute

\section{Source}

National Cancer Institute. CDISC SDTM Observational Study Biospecimen Retention

Terminology. NCl Thesaurus. Code C127258.

Terminology associated with the observational study biospecimen retention codelist of the Clinical Data Interchange Standards Consortium (CDISC) Study Data Tabulation Model (SDT M). 